\title{
Additional Riderships Estimation under Different Configurations of Bus Rapid Transit System
}

\author{
Wu Lan, ${ }^{1}$ Chen Xuewu, ${ }^{2}$ and Lu Tao ${ }^{1}$ \\ ${ }^{1}$ College of Automobile and Traffic Engineering, Nanjing Forestry University, Nanjing 210037, China \\ ${ }^{2}$ School of Transportation, Southeast University, Nanjing 210096, China
}

Correspondence should be addressed to Wu Lan; wulan_nj@163.com

Received 14 January 2015; Accepted 16 February 2015

Academic Editor: Wei (David) Fan

Copyright (C) $2015 \mathrm{Wu}$ Lan et al. This is an open access article distributed under the Creative Commons Attribution License, which permits unrestricted use, distribution, and reproduction in any medium, provided the original work is properly cited.

Different configurations of Bus Rapid Transit (BRT) system may cause different additional riderships. In this paper, in terms of network traffic equilibrium assignment principle, the additional riderships estimation model based on Variational Inequality (VI) model is presented. The bus frequency is related to variables including the travel time, the residence time in terminals, and the dwelling time at the stops. The additional riderships are translated into network additional traffic flow firstly. Given the bus frequency, VI model can be turned into Stochastic User Equilibrium (SUE) model to calculate the other variables. The similarity diagonalization method is used to calculate the elastic bus frequency and finally the network additional traffic flow can be computed. The additional riderships under different configurations of BRT system are compared in the numerical test. The results show that the additional riderships under different configurations have large differences and occupy a high percentage of the total ridership.

\section{Introduction}

Although the foundation ridership can be generated by the increase of the population and the growth of the economy, additional ridership can be generated by Bus Rapid Transit (BRT) system. Different configurations of BRT system induce the diverse scale of additional riderships. Good configurations of BRT system cause the increase of traffic demand and make additional ridership account for a great percentage of the total ridership. The additional riderships induced by different configurations of BRT system are proposed in this paper, which should be considered in the ridership estimation.

The different configurations of BRT system have been attempted by lots of researchers. Laporte et al. [1] reviewed the main optimization methods for the BRT planning. Abdelghany et al. [2] developed a modeling framework for the planning of BRT services in urban transportation network. Aiming at minimizing the total travel time of passengers, Li et al. [3] presented an optimization model for the BRT planning. Schmid [4] proposed a hybrid metaheuristic approach based on large neighborhood search and liner programming to solve the bus rapid transit design problem. In order to capture the effects of BRT services on urban transportation, various methods have been developed, and a majority of those methods are based on simulation. Salem et al. [5] used a CORSIM model to conduct the benefit/cost analysis of the BRT service. Yagi and Mohammadian [6] simulated the BRT development to study the variation of modal split between automobiles and bus rapid transit ridership. Gunawan et al. [7] presented a numerical simulation based on discrete-event approach to identify variables which affects the performance of BRT system. The microscopic simulation techniques are also widely used in the research field of BRT. Yu et al. [8] applied GPS data in the VISSIM-based simulation for BRT system in Beijing. Godavarthi et al. [9] used a microsimulation to find the optimum volume/capacity ratio on BRT routes. Cervero and Kang [10] measured the impacts of implementing BRT on adjacent land usage and land value, by using multilevel models. Based on six characters of BRT system including infrastructure, transport capacity, service level, economic results, safety and emergency management, and energy saving and emission reduction [11], established an evaluation methodology for BRT operation. To study the 
modal shifts to BRT from other modes like automobiles, normal buses, Wang et al. [12] developed a binary logistic analysis approach. Falbel et al. [13] studied the impacts of implementation of BRT on the traffic improvement over the urban transport network, including the additional ridership to the transit system. Currie and Delbosc [14] used several regression models to explore the relationship between BRT design features and increment of ridership. Sun et al. [15] established a numerical model for the headway optimization as well as scheduling combination of BRT vehicles to increase ridership and improve operation performance of BRT. There are few previous literatures that studied the additional ridership under different configurations of BRT system. This paper developed a numerical test based on Variational Inequality (VI) model for additional ridership estimation under different configurations of BRT system. The paper is organized as follows. Section 2 is the problem description. Section 3 is the model development and algorithm for the proposed model. A numerical test is given in Section 4. Section 5 presents the conclusions.

\section{Different Configurations of BRT System Description}

In transportation system, there are many literatures about traffic assignment. Some equilibrium analysis for urban transportation networks were proposed in literature [16, 17]. A traffic equilibrium assignment principle was presented, in the principle, passenger's selection behavior of a transit note was considered. The obtained results showed that the method was effective for network traffic equilibrium assignment [18]. In this paper, the additional riderships estimation under different configurations of BRT system is based on the network traffic equilibrium assignment principle. In terms of the network, the addition ridership account for less than $25 \%$ of the total ridership which is the main restriction in the model [19].

The bus frequency which can reflect the travel time has a function relation with the traffic flow on the road link [20]. It is the main variable in the model, which can represent the requirements of the different hardware configurations and the standards of operational service.

The traffic flow on the road link of BRT system is a function of the bus frequency, which is determined by the bus operation cycle and the bus number of each line. $t_{m}^{l}$ denotes the travel time on the road link $m$ of the bus route $l$, and the bus operation cycle $T_{l}$ is determined by the traffic conditions of network and the dwelling time at the stop $d_{n}^{l}$ :

$$
T_{l}=\rho t_{0}^{l}+\sum_{m \in l} t_{m}^{l}+\sum_{n \in l} d_{n}^{l}
$$

where $m \in l$ and $n \in l$ represent that the road link $m$ and transfer stop $n$ belong to the bus route $l$, respectively. $t_{0}^{l}$ represents the residence time in the terminal. $\rho$ denotes the number of terminals and $\rho=1$ indicates there is only a terminal, while $\rho=2$ indicates there are two terminals.
TABLE 1: The variation range of $t_{m}^{l}$ for 1 kilometer in Changzhou BRT.

\begin{tabular}{lc}
\hline Configuration level & Travel time for $1 \mathrm{~km} \mathrm{(s)}$ \\
\hline Primary & $146 \sim 215$ \\
Intermediate & $120 \sim 144$ \\
Advanced & $90 \sim 144$
\end{tabular}

$N_{l}$ denotes the bus number of the route $l$; then bus frequency of the route $l$ can be calculated with the following equation:

$$
f_{l}=\frac{N_{l}}{\rho t_{0}^{l}+\sum_{m \in l} t_{m}^{l}+\sum_{n \in l} d_{n}^{l}} .
$$

The travel time $t_{m}^{l}$ has a relationship with adopting signal priority control and the configurations of route. If the signal priority control is adopted, the intersection delay and the travel time will be shortened. When the route is a part of BRT system, the security and the speed of driving will increase. According to the travel time survey in Changzhou BRT, for 1 kilometer section, the variation range of the travel time $t_{m}^{l}$ of different configurations is as shown in Table 1.

If the bus frequency is held constant, the residence time in the terminal $t_{0}^{l}$ is related to the number of buses on the route. And the existence of more buses means longer residence time in the terminal. In terms of the BRT system under different configurations, the bus capacity varies with the bus model. For the convenience of calculation and comparison, the different BRT bus models are replaced by a standard BRT bus model, the size of which is $12 \mathrm{~m}$, and the conversion coefficient is the ratio of bus capacity.

The dwelling time at a stop $d_{n}^{l}$ is consisted of the time of deceleration and acceleration, the time of switching door, and the time of boarding and alighting. When the bus model, the platform, and the lane are constant, the dwelling time at a stop is determined by the number of the passengers boarding and alighting. The boarding and alighting time are determined by passenger number and the average time of per boarding and alighting passenger $w_{\text {on }}, w_{\text {off }}$, respectively. Wirasinghe and Szplett [21] presented that if the given road link traffic flow is $\mathbf{v}$, the number of passengers alighting and boarding at the stop $n$ on the bus route $l$ can be yielded as follows:

$$
\begin{aligned}
& \mathrm{Al}_{n}^{l}=\sum_{s \in S} \delta_{n s}^{-} \xi_{l s} x_{s}^{l} \mathbf{v}_{s}, \\
& \mathrm{Bo}_{n}^{l}=\sum_{s \in S} \delta_{n s}^{+} \xi_{l s} x_{s}^{l} \mathbf{v}_{s},
\end{aligned}
$$

where $\mathrm{Al}_{n}^{l}$ and $\mathrm{Bo}_{n}^{l}$ denote the number of passengers alighting and boarding at the stop $n$ on the bus route $l$, respectively. $w_{\text {on }}$ and $w_{\text {off }}$ denote the average time of per boarding and alighting passenger, respectively. Thus, $w_{\text {on }} \mathrm{Bo}_{n}^{l}$ denotes the boarding time and $w_{\text {off }} \mathrm{Al}_{n}^{l}$ denotes the alighting time at the stop $n$. $x_{s}^{l}$ denotes the percentage of riderships who choose route $l$ on the road link $s$, and $v_{s}$ denotes the ridership on the road link $s . \delta_{n s}^{+}, \delta_{n d}^{-}$, and $\xi_{l s}$ are defined as exponential functions, where the superscripts with plus sign and minus sign indicate the 
departure terminal and the destination terminal on the road link $s$. The dwelling time at the stop $n$ can be yielded as follows:

$$
d_{n}^{l}=d_{\mathrm{de}}^{l}+w_{\mathrm{off}} \mathrm{Al}_{n}^{l}+w_{\mathrm{on}} \mathrm{Bo}_{n}^{l}+d_{\mathrm{ac}}^{l}+d_{\mathrm{door}}^{l}
$$

where $d_{\mathrm{de}}^{l}$ denotes the time of deceleration, which means the time from vehicle slowing down to a full stop. $d_{\mathrm{ac}}^{l}$ denotes the time of acceleration, which means the time from starting acceleration to moving in a steady speed. $d_{\text {door }}^{l}$ denotes the time of opening and closing door. Passengers alighting are assumed to be completed before passengers boarding at the stop. The main factors influencing the dwelling time at a stop $d_{n}^{l}$ are the average time of per boarding and alighting passenger $w_{\text {on }}, w_{\text {off }}$, respectively. The average time of per boarding and alighting passenger is influenced by fare collection method, the density of passengers inside and outside the vehicle, width of the bus door, whether to keep the platform and the vehicle floor in the same horizontal plane, and other uncertain conditions like weather.

Thus, the bus frequency varies with the different configurations of BRT system, which reflects the hardware configuration and the operation state significantly. When the vehicle model, the running ways form, and the platform style are fixed, the dwelling time of a vehicle can be presented as a function of the traffic flow $\mathbf{v}$ as shown in formula (5). $\mathbf{v}$ denotes the traffic flow on the road link in the public transportation network, which can be transformed to ridership based on the average capacity and the vehicle conversion coefficient

$$
d_{n}^{l}=d_{n}^{l}(\mathbf{v})
$$

When the configuration is determined, bus frequency is also a function of the traffic flow $\mathbf{v}$; the elastic bus frequency can be yielded as follows:

$$
f_{l}=\frac{N_{l}}{T_{l(\mathbf{v})}}
$$

where $N_{l}$ denotes the bus number of the route $l$ and $T_{l}(\mathbf{v})$ is the operation cycle related to the traffic flow on bus line $l$. $v$ is the traffic flow in the transit network. The traffic flow can be converted to ridership by bus capacity and vehicle conversion coefficient.

\section{The Additional Riderships Estimation Based on VI Model}

In the model, the additional ridership is translated into additional traffic flow in the network $\sum_{i} o_{i}$. The relationship between traffic generation and traffic distribution can be represented as follows:

$$
\sum_{j \in J} d_{i j}=o_{i}, \quad i \in I
$$

where $d_{i j}$ denotes the additional traffic distribution. The relationship between the traffic distribution and the path flow can be formularized as follows:

$$
\sum_{r \in R_{i j}} h_{r}^{i j}=d_{i j}, \quad i \in I, \quad j \in j,
$$

where $h_{r}^{i j}$ denotes the route flow between $i$ and $j$ on route $r$. The relationship between the traffic flow $\mathbf{v}$ in the network and the path flow can be defined as follows:

$$
\mathbf{v}=\mathbf{A}(\overline{\mathbf{h}}+\mathbf{h})
$$

where $\mathbf{A}$ denotes an incidence matrix regarding road linkpath. If the road link $s$ is on path $r$, it is equal to 1 ; otherwise it equals $0 . \overline{\mathbf{h}}$ denotes the path flow driven by the current traffic demand, while $\mathbf{h}$ denotes the additional path flow driven by the additional traffic demand. Thus,

$$
f_{l}=\frac{N_{l}}{T_{l}(\mathbf{A}(\overline{\mathbf{h}}+\mathbf{h}))}=\frac{N_{l}}{T_{l}^{\prime}(\overline{\mathbf{h}}, \mathbf{h})},
$$

where $N_{l}$ represents the number of buses on the route $l$, which is often held constant:

$$
f_{l}=f_{l}(\overline{\mathbf{h}}, \mathbf{h})
$$

The passenger flow at the stop is a function of the bus frequency. It makes it hard to obtain the Karush-Kuhn-Tucker (KKT) condition of the Stochastic User Equilibrium (SUE) model [22] and leads to the difficulty of solving the model. When a variable (such as bus frequency) is held constant, the VI model is used to avoid this problem. The VI model can be transformed to the UE optimization model, which has a unique solution.

3.1. VI Model Formulation. It has been proved that the network equilibrium model and the VI model are equivalent. Assuming that the traffic cost $t_{r}^{i j}>0$ and the additional traffic demand $d_{i j} \geq 0$, let $\mathbf{x}=\left(\overline{\mathbf{h}}^{n_{1}}, \mathbf{h}^{n_{2}}, \mathbf{d}^{n_{3}}, \mathbf{f}^{n_{4}}\right)^{T}$, where $\mathbf{x} \in R^{n}$ is a vector of parameters; $\overline{\mathbf{h}}$ denotes the path flow driven by the current traffic demand; $\mathbf{h}$ denotes the additional path flow driven by the additional traffic demand; d denotes the additional traffic distribution; $\mathbf{f}$ is the bus frequency vector of route. According to the SUE model, a continuous function $F$ 
of each vector variable can be referred to as formula (12) by the letters $\mathbf{A}, \mathbf{B}, \mathbf{C}$, and $\mathbf{D}$ in vector:

$$
\begin{aligned}
& F\left(\overline{\mathbf{h}}^{n_{1}^{*}}\right)=\left(F_{1}^{i j}\left(\bar{h}^{1 *}\right), F_{2}^{i j}\left(\bar{h}^{2 *}\right), \ldots, F_{n_{1}}^{i j}\left(\bar{h}^{n_{1} *}\right)\right)^{T} \\
& =\left(\frac{1}{\theta_{1}} \ln h_{1}^{i j *}+\sum_{s \in S}\left(t_{s}+u_{s}\left(f^{*}\right)\right) \delta_{s 1}^{i j}, \frac{1}{\theta_{1}} \ln h_{2}^{i j *}\right. \\
& +\sum_{s \in S}\left(t_{s}+u_{s}\left(f^{*}\right)\right) \delta_{s 2}^{i j}, \ldots, \frac{1}{\theta_{1}} \ln h_{3}^{i j *} \\
& \left.+\sum_{s \in S}\left(t_{s}+u_{s}\left(f^{*}\right)\right) \delta_{s 3}^{i j}\right)^{T}=\mathbf{A} \\
& F\left(\mathbf{h}^{n_{2}^{*}}\right)=\left(F_{1}^{i j}\left(h^{1 *}\right), F_{2}^{i j}\left(h^{2 *}\right), \ldots, F_{n_{2}}^{i j}\left(h^{n_{2} *}\right)\right)^{T} \\
& =\left(\frac{1}{\theta_{2}} \ln h_{1}^{i j *}+\sum_{s \in S}\left(t_{s}+u_{s}\left(f^{*}\right)\right) \delta_{s 1}^{i j}, \frac{1}{\theta_{2}} \ln h_{2}^{i j *}\right. \\
& +\sum_{s \in S}\left(t_{s}+u_{s}\left(f^{*}\right)\right) \delta_{s 2}^{i j}, \ldots, \frac{1}{\theta_{2}} \ln h_{3}^{i j *} \\
& \left.+\sum_{s \in S}\left(t_{s}+u_{s}\left(f^{*}\right)\right) \delta_{s 3}^{i j}\right)^{T}=\mathbf{B} \\
& F\left(\mathbf{d}^{n_{3}^{*}}\right) \\
& =\left(F_{1}\left(d^{1 *}\right), F_{2}\left(d^{2 *}\right), \ldots, F_{n_{3}}\left(d^{n_{3} *}\right)\right)^{T} \\
& =\left(\frac{1}{\theta_{2}} \ln d_{i j}^{*}+c_{j}, \frac{1}{\theta_{2}} \ln d_{i j}^{*}+c_{j}, \ldots, \frac{1}{\theta_{2}} \ln d_{i j}^{*}+c_{j}\right)^{T}=\mathbf{C}, \\
& F\left(\mathbf{f}^{n_{2}}\right)=(0,0, \ldots, 0)^{T}=\mathbf{D},
\end{aligned}
$$

where $\theta_{1}, \theta_{2}>0, i \in I, j \in J$.

The VI model can be expressed as follows:

$$
\begin{aligned}
& F\left(\mathbf{x}^{*}\right)^{T}\left(\mathbf{x}-\mathbf{x}^{*}\right) \\
& \quad=(\mathbf{A}, \mathbf{B}, \mathbf{C}, \mathbf{D})\left(\overline{\mathbf{h}}-\overline{\mathbf{h}}^{*}, \mathbf{h}-\mathbf{h}^{*}, \mathbf{d}-\mathbf{d}^{*}, \mathbf{f}-\mathbf{f}^{*}\right) \geq 0,
\end{aligned}
$$

where there is a constraint $\Omega=\{G(\mathbf{x}) \geq 0, H(\mathbf{x})=0\}$. In formula (12), $t_{s}$ represents the travel cost on the road link $s ; u_{s}^{i j}$ denotes the user waiting time on the road link $s$ and $c_{j}$ denotes the destination attractive function. The passenger waiting time on the road link $s$ is determined as $u_{s}=\alpha / f_{s} . f_{s}$ denotes the bus frequency on the road link $s$ and is equal to the bus frequency of route $l, f_{l}$. The parameter $\alpha$ is determined by the distribution value of bus headway, where $\alpha=1$ represents an exponential distribution and $\alpha=0.5$ represents a uniform distribution [23]. $\alpha=0.5$ is commonly used in the practical analysis.

Assuming that $x^{*}$ is a solution of the VI model (13) and the linear independence condition is satisfied at $\left(x^{*}\right)$, then the GKKT (General Karush-Kuhn-Tucker) conditions are satisfied.
The constraint $H(\mathbf{x})=0$ can be yielded as follows:

$$
\begin{gathered}
\sum_{j \in J} d_{i j}=o_{i}, \quad i \in I, \\
\sum_{r \in R_{i j}} \bar{h}_{r}^{i j}=\bar{d}_{i j}, \quad i \in I, \quad j \in J, \\
\sum_{r \in R_{i j}} h_{r}^{i j}=d_{i j}, \quad i \in I, \quad j \in J,
\end{gathered}
$$

and the constraint $G(\mathbf{x}) \geq 0$ can be yielded as follows:

$$
\begin{gathered}
0 \leq \frac{\sum_{i} o_{i}}{\left(\sum_{i} \bar{o}_{i}+\sum_{i} o_{i}\right)} \leq \gamma, \\
\bar{h}_{r}^{i j} \geq 0, \quad i \in I, \quad j \in J, \quad r \in R_{i j}, \\
h_{r}^{i j} \geq 0, \quad i \in I, \quad j \in J, \quad r \in R_{i j}, \\
d_{i j} \geq 0, \quad i \in I, \quad j \in J, \\
0 \leq f \leq 60 .
\end{gathered}
$$

Formula (17) is based on the condition that the addition ridership accounts for less than a percent of the total ridership. According to the TCRP118, the percentage was suggested as $25 \%$.

In formula (21), the part " $f \leq 60$ " is based on the practices. Usually the time interval of two consecutive buses is in the range of $1 \mathrm{~min}$ to $6 \mathrm{~min}$; thus $f_{\max }=1 /(1 / 60)=60 \mathrm{veh} / \mathrm{h}$. The GKKT conditions are defined as follows:

$$
\begin{gathered}
\frac{1}{\theta_{1}} \ln \bar{h}_{r}^{i j *}+\sum_{s}\left(t_{s}+u_{s}\left(f^{*}\right)\right) \delta_{s r}^{i j}-v_{2}^{i j *}=0, \\
\frac{1}{\theta_{2}} \ln h_{r}^{i j *}+\sum_{s}\left(t_{s}+u_{s}\left(f^{*}\right)\right) \delta_{s r}^{i j}-v_{3}^{i j *}=0, \\
\frac{1}{\theta_{2}} \ln d_{i j}^{*}-v_{1}^{i *}+v_{3}^{i j *}+c_{j}=0,
\end{gathered}
$$

where, $i \in I, j \in J, r \in R$.

By invoking (14), (16) is solved as follows:

$$
d_{i j}^{*}=o_{i} \frac{\exp \left[-\theta_{2}\left(v_{3}^{i j *}+c_{j}\right)\right]}{\sum_{j \in J} \exp \left[-\theta_{2}\left(v_{3}^{i j *}+c_{j}\right)\right]} .
$$

Let $t_{r}^{i j}=\sum_{s} t_{s}^{i j} \delta_{s r}^{i j}$ present the travel cost on route $r$ and $u_{r}^{i j}=$ $\sum_{s} u_{s}^{i j} \delta_{s r}^{i j}$ present the user waiting time on route $r$. Equations (15) and (16) can be derived as follows:

$$
\begin{aligned}
& \bar{h}_{i j}^{*}=\bar{d}_{i j} \frac{\exp \left[-\theta_{1}\left(t_{r}^{i j}+u_{r}^{i j}\right)\right]}{\sum_{k \in R_{i j}} \exp \left[-\theta_{1}\left(t_{k}^{i j}+u_{k}^{i j}\right)\right]}, \\
& h_{i j}^{*}=d_{i j} \frac{\exp \left[-\theta_{2}\left(t_{r}^{i j}+u_{r}^{i j}\right)\right]}{\sum_{k \in R_{i j}} \exp \left[-\theta_{2}\left(t_{k}^{i j}+u_{k}^{i j}\right)\right]},
\end{aligned}
$$

where $P_{r}^{i j}=\exp \left[-\theta_{2}\left(t_{r}^{i j}+u_{r}^{i j}\right)\right] / \sum_{k \in R_{i j}} \exp \left[-\theta_{2}\left(t_{k}^{i j}+u_{k}^{i j}\right)\right]$ is the logit model based on the path selection probability. 
TABLE 2: Road link information data of the transit network.

\begin{tabular}{|c|c|c|c|c|c|c|c|c|c|c|}
\hline \multirow{2}{*}{ Road link data } & \multicolumn{10}{|c|}{ Road link } \\
\hline & $L_{1}^{1}$ & $L_{1}^{2}$ & $L_{1}^{3}$ & $L_{2}^{1}$ & $L_{2}^{2}$ & $L_{3}$ & $L_{4}$ & $L_{5}$ & $L_{6}$ & $L_{7}$ \\
\hline $\mathrm{Nl}$ (standard BRT bus model) & 19 & 19 & 19 & 20 & 20 & 11 & 11 & 30 & 30 & 12 \\
\hline Travel time (min) & 16 & 18 & 20 & 13 & 15 & 13 & 16 & 31 & 35 & 24 \\
\hline
\end{tabular}

3.2. Solution Algorithm. When $f$ is given, let $F=\nabla g$; the objective function $g$ can be written as follows:

$$
\begin{aligned}
\min g\left(h_{r}^{i j}, d_{i j}\right)= & \sum_{s \in S} \int_{0}^{\sum_{s \in S} h_{r}^{i j} \delta_{s r}^{i j}}\left(t_{s}+u_{s}\right) d v \\
& +\sum_{j \in J} \int_{0}^{\sum_{i \in I} d_{i j}} c_{j}(y) d y \\
& +\frac{1}{\theta_{1}} \sum_{i \in I} \sum_{j \in J} \sum_{r \in R} h_{r}^{i j}\left(\ln h_{r}^{i j}-1\right) \\
& +\frac{1}{\theta_{2}} \sum_{i \in I} \sum_{j \in J} d_{i j}\left(\ln d_{i j}-1\right)
\end{aligned}
$$

which subjects to constraints (14)-(21). This model is a SUE assignment problem and satisfies the KKT conditions.

In public transport problems, the passenger travel time is independent of the traffic flow [24-27]. Once the bus frequency is certain, the expected passenger travel time $C_{r}^{i j}$ is independent of the path flow and the bus frequency:

$$
C_{r}^{i j}=t_{r}^{i j}+u_{r}^{i j}+\xi_{r}^{i j}, \quad i \in I, j \in J, r \in R_{i j} .
$$

$t_{r}^{i j}$ denotes the travel time at the road link $r . u_{r}^{i j}$ indicates the waiting time at the stop, and $\xi_{r}^{i j}$ is the random error. As long as $f$ is fixed, the optimization problem (25) can be solved without iteration. Meanwhile, the VI model can be transformed into the optimization SUE model to solve the other two variables $h$ and $d$. This characteristic can be used in the similarity diagonalization method to iterate and solve the VI model based on the elastic frequency.

\section{Numerical Test}

4.1. Network Basic Information. In order to demonstrate the performance of the proposed model and solution algorithms, numerical tests were carried out on a real transit network in Changzhou city (see Figure 1). In this study, Wujin district (node 1) and New North district (node 2) are considered as the origins, while Jin Ling road (node 3 ) and River road (node 4 ) are considered as the destinations. $L_{1}^{1}$ (from node 1 to node 5 ), $L_{1}^{2}$ (from node 5 to node 6 ), and $L_{1}^{3}$ (from node 6 to node 3 ) are road links of route $L_{1}$, while $L_{2}^{1}$ and $L_{2}^{2}$ are the road links of route $L_{2}$.

The road link information of the transit network shown in Figure 1 is given in Table 2. $L_{2}, L_{5}$, and $L_{6}$ are BRT routes. The traffic demand is shown in Table 3.
TABLE 3: The OD travel matrix of the transit network.

\begin{tabular}{lccc}
\hline OD $($ pass $/ \mathrm{h})$ & 3 & 4 & $\Sigma$ \\
\hline 1 & 2000 & 1000 & 3000 \\
2 & 500 & 1500 & 2000 \\
\hline$\Sigma$ & 2500 & 2500 & \\
\hline
\end{tabular}

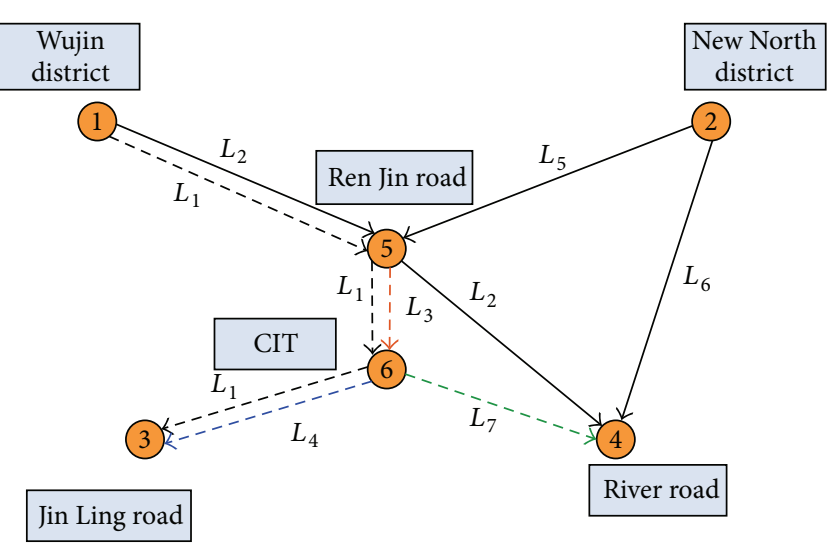

$\longrightarrow L_{2}, L_{5}, L_{6}$-: BRT lines $\quad-\rightarrow L_{4}$-: number 29 bus line

$\rightarrow L_{1}-$ : number 33 bus line $\quad-\rightarrow L_{7}-$ : number 40 bus line

$-\rightarrow L_{3}-$ : number 14 bus line

FIGURE 1: A portion of transit network of Changzhou city.

The dwelling time at a stop under different configurations can be yielded as follows:

primary configuration:

$$
d_{n}^{l}=\frac{12+2.58\left(\mathrm{Al}_{n}^{l}+\mathrm{Bo}_{n}^{l}\right)}{3600}
$$

intermediate configuration:

$$
d_{n}^{l}=\frac{12.33 \times 0.9+12 \times 0.1+1.51\left(\mathrm{Al}_{n}^{l}+\mathrm{Bo}_{n}^{l}\right)}{3600}
$$

advanced configuration:

$$
d_{n}^{l}=\frac{13 \times 0.9+12 \times 0.1+0.72\left(\mathrm{Al}_{n}^{l}+\mathrm{Bo}_{n}^{l}\right)}{3600}
$$

the dwelling time for a regular bus:

$$
d_{n}^{l}=\frac{10+2.58\left(\mathrm{Al}_{n}^{l}+\mathrm{Bo}_{n}^{l}\right)}{3600}
$$




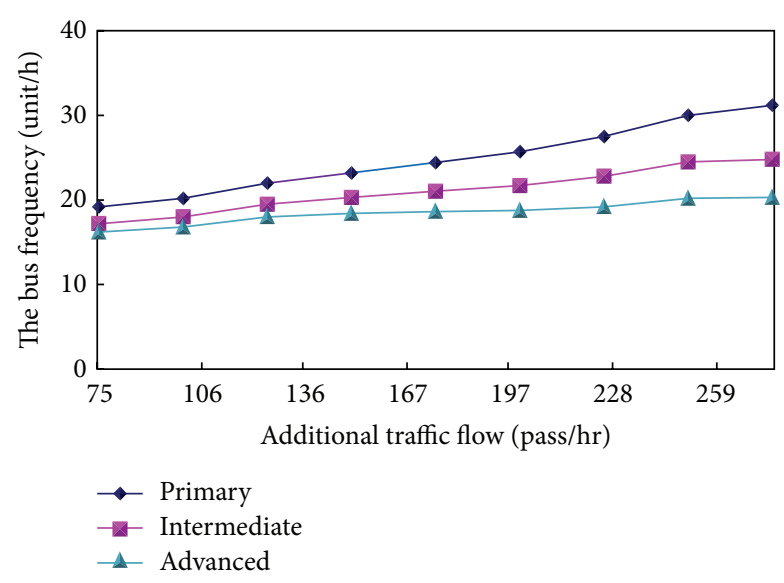

FIGURE 2: The change of the bus frequency under different configurations of BRT line 1.

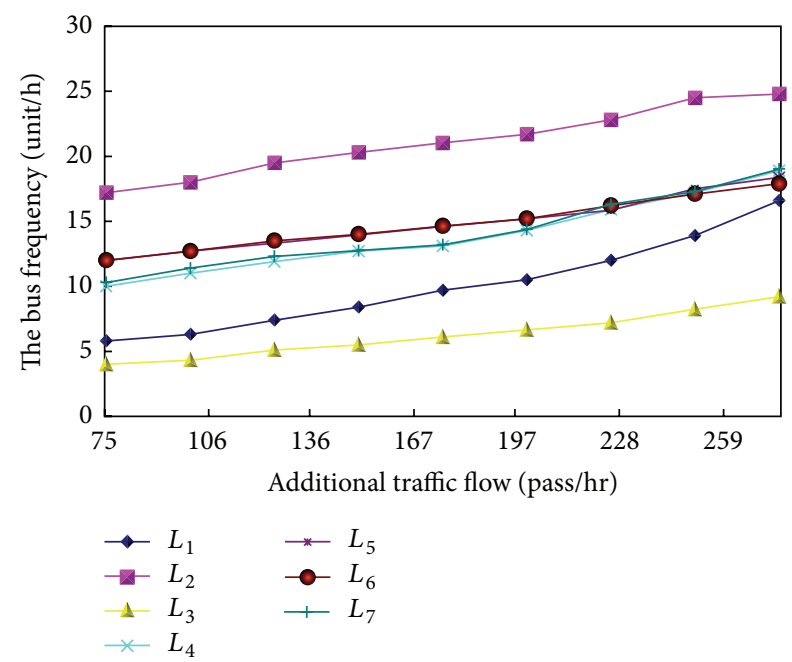

FIGURE 3: The change of the bus frequency with the increase of traffic flow.

4.2. Results. Through the numerical test, it is concluded that the bus frequency under different configurations of BRT line 1 ( $L_{2}$ in the network) increases with the traffic flow. The increase of the bus frequency under primary configuration is bigger than that under advanced configuration (Figure 2).

Figure 3 shows the change of the bus frequency on each route with the increase of traffic flow, where the BRT system is under intermediate configuration. The bus frequency variation of BRT lines $\left(L_{2}, L_{5}\right.$, and $\left.L_{6}\right)$ is more uniform than that of common ones $\left(L_{1}, L_{3}, L_{4}\right.$, and $\left.L_{7}\right)$. The bus frequency of $L_{1}$ is the most sensitive to the additional traffic flow, while the frequency of $L_{3}$ is lower than others because of the shorter travel distance. In addition, Figure 4 shows the additional traffic flow occupy. From Figure 4, it can be found that the total traffic flow increases obviously and the percentages of total traffic flow under different configurations are $11.9 \%$, $18.8 \%$ and $22.8 \%$, respectively compared with the original traffic flow $(5000 / \mathrm{h})$. The additional traffic flow occupies a percentage of total traffic flow that is $11.9 \%, 18.8 \%$, and $22.8 \%$, respectively.

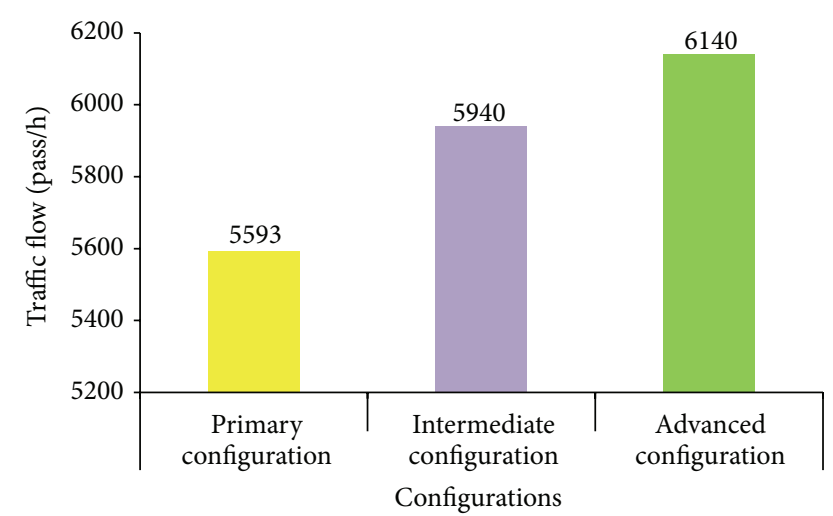

Figure 4: Traffic flow under different configurations.

\section{Conclusions}

In this study, the additional riderships prediction method based on VI model under different BRT system configuration are presented, as well as the solution algorithm. The VI model is turned into the SUE model, in order to avoid the solving difficulty resulted from the elastic frequency. A transform between the VI model and the UE model is proposed in the solution, where the similarity diagonalization method can be used. The additional traffic flow under different configurations of BRT system is computed in the numerical test. The results show that the potential riderships of different configurations are obviously different, and the bus frequency under primary configuration has the largest increase with the rise of network traffic flow. It also can be seen that the potential riderships have a big proportion in the total ridership.

Considering those differences in the construction of BRT, different elements of the system should be chosen in different development stages to achieve the objective of TOD, in addition to saving the cost of investments.

\section{Conflict of Interests}

The authors declare that there is no conflict of interests regarding the publication of this paper.

\section{Acknowledgment}

This work is supported by National Natural Science Foundation of China 51408314.

\section{References}

[1] G. Laporte, J. A. Mesa, and F. A. Ortega, "Optimization methods for the planning of rapid transit systems," European Journal of Operational Research, vol. 122, no. 1, pp. 1-10, 2000.

[2] K. F. Abdelghany, H. S. Mahmassani, and A. F. Abdelghany, "A modeling framework for bus rapid transit operations evaluation and service planning," Transportation Planning and Technology, vol. 30, no. 6, pp. 571-591, 2007. 
[3] J. Q. Li, M. Song, M. Li, and W. B. Zhang, "Planning for bus rapid transit in single dedicated bus lane," Transportation Research Record, vol. 2111, no. 1, pp. 76-82, 2009.

[4] V. Schmid, "Hybrid large neighborhood search for the bus rapid transit route design problem," European Journal of Operational Research, vol. 238, no. 2, pp. 427-437, 2014.

[5] O. Salem, H. Wei, and V. Iragavarapu, "Impact of BRT on Martin Luther King Dr and Madison Road," in Proceedings of the IEEE Intelligent Transportation Systems Conference (ITSC '06), pp. 6167, Toronto, Canada, September 2006.

[6] S. Yagi and A. Mohammadian, "Policy simulation for new BRT and area pricing alternatives using an opinion survey in Jakarta," Transportation Planning and Technology, vol. 31, no. 5, pp. 589612, 2008.

[7] F. E. Gunawan, Suharjito, and A. A. S. Gunawan, "Simulation model of bus rapid transit," EPJ Web of Conferences, vol. 68, Article ID 00021, 2014.

[8] L. Yu, L. Yu, X. Chen, T. Wan, and J. F. Guo, "Calibration of VISSIM for bus rapid transit systems in Beijing using GPS data," Journal of Public Transportation, vol. 9, no. 3, pp. 239-257, 2006.

[9] G. R. Godavarthi, R. S. Chalumuri, and S. Velmurugun, "Measuring the performance of bus rapid-transit corridors based on volume by capacity ratio," Journal of Transportation Engineering, vol. 140, no. 10, Article ID 04014049, 2014.

[10] R. Cervero and C. D. Kang, "Bus rapid transit impacts on land uses and land values in Seoul, Korea," Transport Policy, vol. 18, no. 1, pp. 102-116, 2011.

[11] H. Y. Wu, "Evaluation methodology of bus rapid transit (BRT) operation," Journal of Theoretical and Applied Information Technology, vol. 48, no. 3, pp. 1733-1737, 2013.

[12] Y. Wang, Z. Wang, Z. Li, S. R. Staley, A. T. Moore, and Y. Gao, "Study of modal shifts to bus rapid transit in Chinese cities," Journal of Transportation Engineering, vol. 139, no. 5, pp. 515523, 2013.

[13] S. Falbel, P. Rodriguez, H. Levinson et al., "Bus rapid transit plans in New York's capital district," Journal of Public Transportation, vol. 9, no. 3, pp. 23-50, 2006.

[14] G. Currie and A. Delbosc, "Understanding bus rapid transit route ridership drivers: an empirical study of Australian BRT systems," Transport Policy, vol. 18, no. 5, pp. 755-764, 2011.

[15] C.-J. Sun, W. Zhou, and Y.-Q. Wang, "Scheduling combination and headway optimization of bus rapid transit," Journal of Transportation Systems Engineering and Information Technology, vol. 8, no. 5, pp. 61-67, 2008.

[16] Y. Sheffi, Urban Transportation Networks: Equilibrium Analysis with Mathematical Programming Methods, Prentice-Hall, Englewood Cliffs, NJ, USA, 1985.

[17] B. Z. Yao, P. Hu, X. H. Lu, J. J. Gao, and M. H. Zhang, "Transit network design based on travel time reliability," Transportation Research Part C: Emerging Technologies, vol. 43, pp. 233-248, 2014.

[18] B. Yu, H. Zhu, W. Cai, N. Ma, Q. Kuang, and B. Yao, "Twophase optimization approach to transit hub location-the case of Dalian," Journal of Transport Geography, vol. 33, pp. 62-71, 2013.

[19] Kittelson \& Associates, "Bus rapid transit practitioner's guide," TCRP Report 118, Trasportation Research Board of the National Academies, Washington, DC, USA, 2007.

[20] B. Yu, Z. Z. Yang, X. S. Sun, B. Z. Yao, Q. C. Zeng, and E. Jeppesen, "Parallel genetic algorithm in bus route headway optimization," Applied Soft Computing Journal, vol. 11, no. 8, pp. 5081-5091, 2011.
[21] S. C. Wirasinghe and D. Szplett, "An investigation of Passenger interchange and train standing time at LRT stations: (II) estimation of standing time," Journal of Advanced Transportation, vol. 18, no. 1, pp. 13-24, 1984.

[22] Q. Meng, L. Wu, and D. H. Lee, "Maximal reserve trip generation for transit networks," Journal of the Eastern Asia Society for Transportation Studies, vol. 7, pp. 570-585, 2008.

[23] W. H. K. Lam, J. Zhou, and Z.-H. Sheng, "A capacity restraint transit assignment with elastic line frequency," Transportation Research Part B: Methodological, vol. 36, no. 10, pp. 919-938, 2002.

[24] B. Z. Yao, P. Hu, M. H. Zhang, and M. Q. Jin, "A support vector machine with the tabu search algorithm for freeway incident detection," International Journal of Applied Mathematics and Computer Science, vol. 24, no. 2, pp. 397-404, 2014.

[25] B. Z. Yao, P. Hu, M. H. Zhang, and X. M. Tian, "Improved ant colony optimization for seafood product delivery routing problem," PROMET-Traffic \& Transportation C, vol. 26, no. 1, pp. $1-10,2014$.

[26] B. Z. Yao, B. Yu, J. J. Gao, and M. H. Zhang, "An improved particle swarm optimization for carton heterogeneous vehicle routing problem with a collection depot," Annals of Operations Research, 2014.

[27] B. Yao, P. Hu, M. Zhang, and S. Wang, "Artificial bee colony algorithm with scanning strategy for the periodic vehicle routing problem," Simulation, vol. 89, no. 6, pp. 762-770, 2013. 


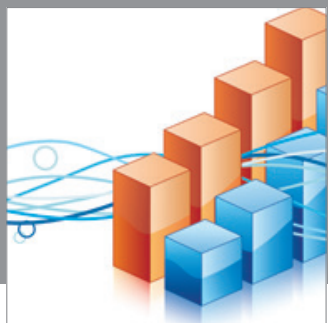

Advances in

Operations Research

mansans

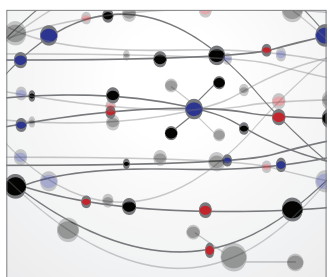

The Scientific World Journal
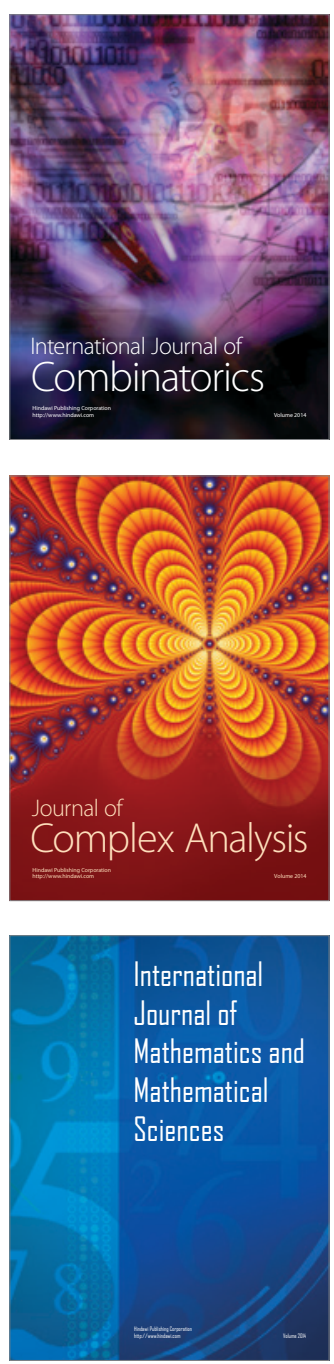
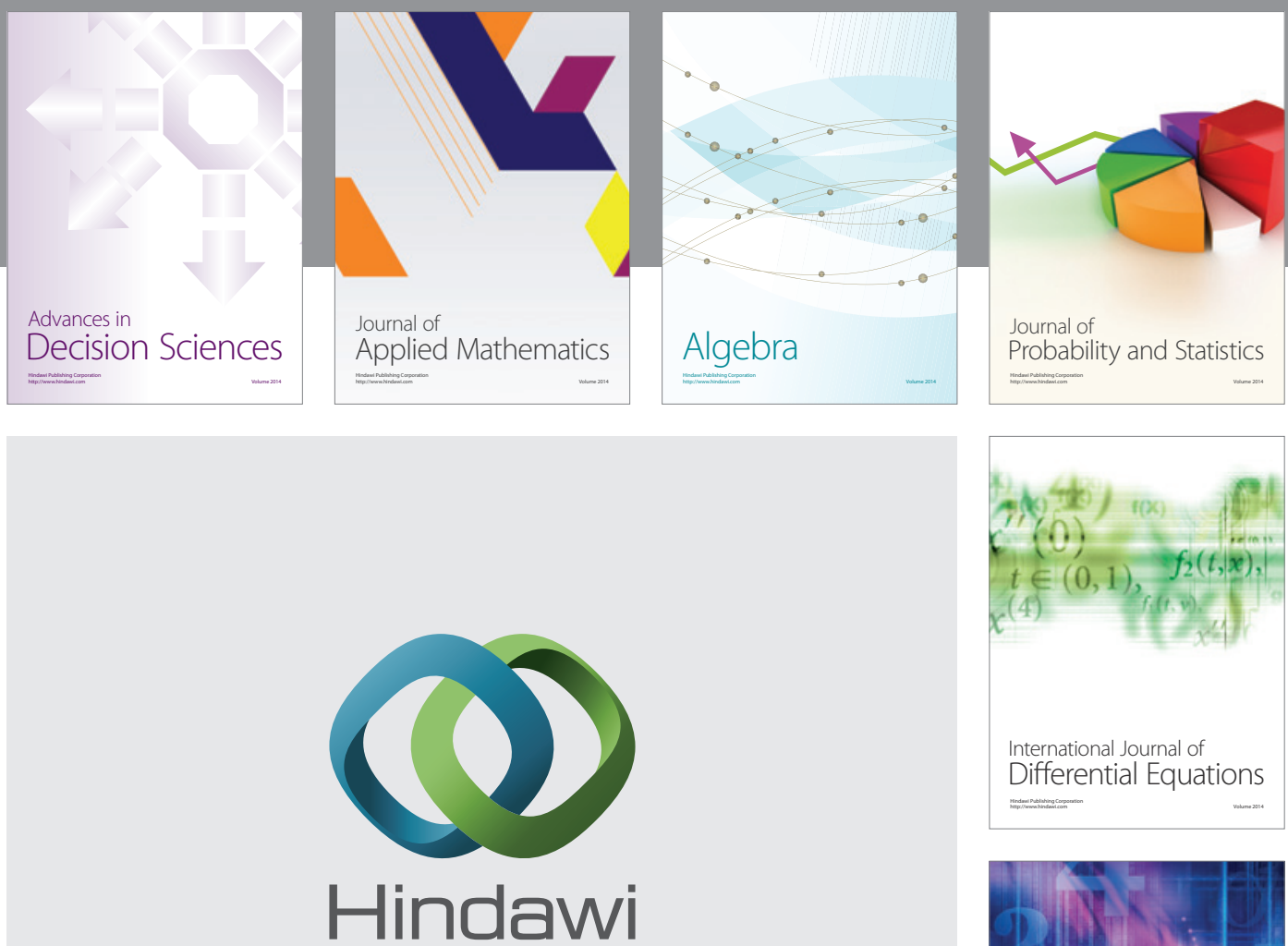

Submit your manuscripts at http://www.hindawi.com
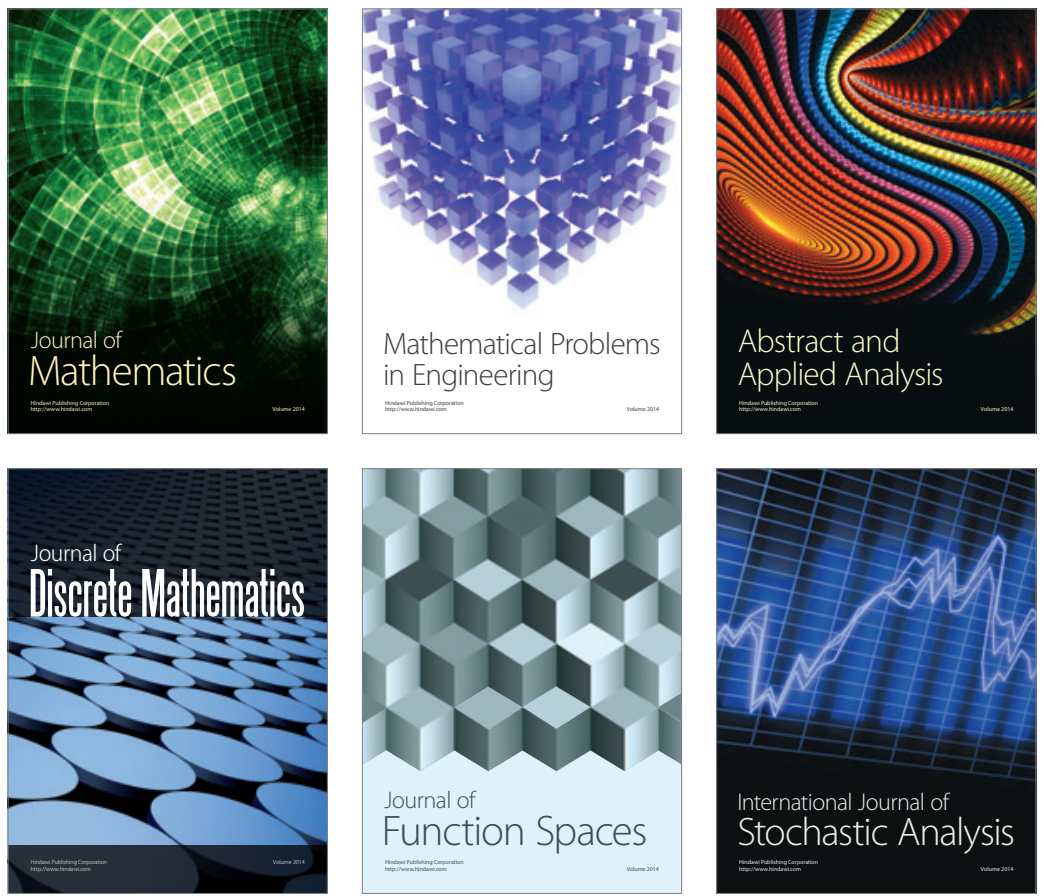

Journal of

Function Spaces

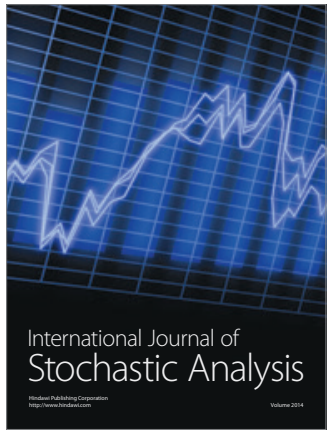

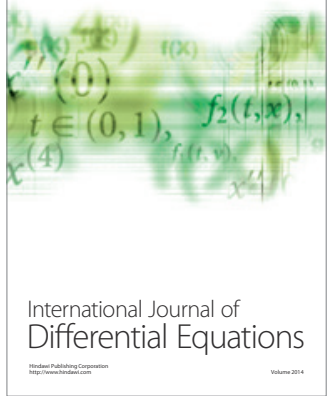
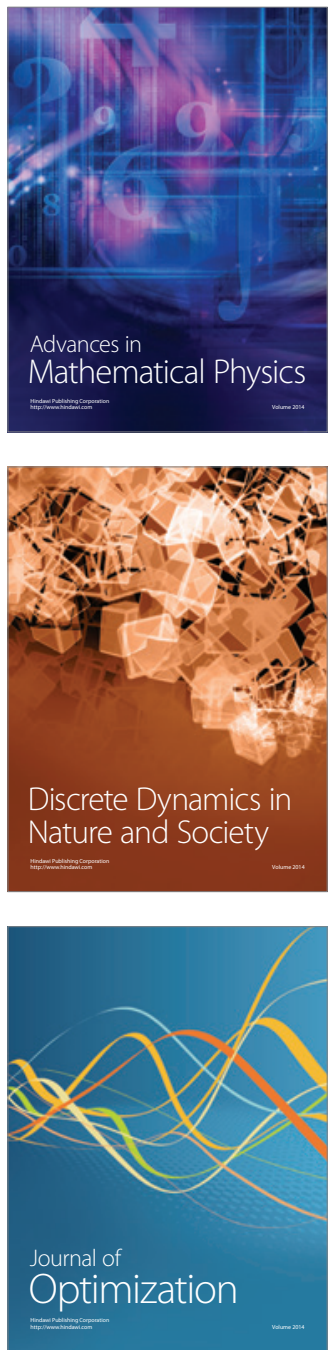